DOI: $10.29184 / 1980-7813 . r c f m c .181 . v o l .12 . n 3.2017$

\title{
SINAIS DE ALERTA PARA TRANSTORNO DO ESPECTRO DO AUTISMO EM CRIANÇAS DE 0 A 3 ANOS
}

\section{ALERTS FOR AUTISM SPECTRUM DISORDERS IN CHILDREN FROM 0 TO 3 YEARS}

\author{
Odila Maria F. Carvalho Mansur¹, Leila Regina d'Oliveira de Paula Nunes ${ }^{2}$, Adriana Fantoni Naurath Colares ${ }^{3}$ \\ Brígida Maria Pereira Barbosa da Silva ${ }^{4}$, Laura Carvalho Mansur ${ }^{5}$
}

1- Pedagoga do Instituto Federal Fluminense, Professora de Pediatria da Faculdade de Medicina de Campos (FMC), Mestre em Cognição e Linguagem (UENF), Doutoranda em Educação Especial (UERJ).

2- Psicóloga; Professora da Universidade do Estado do Rio de Janeiro-RJ (UERJ), Doutorado (PhD) em Especial Education na Vanderbilt University-EUA.

3 e 4- Psicopedagogas, alunas do Curso de Extensão em Perinatologia, da Faculdade de Medicina de Campos (FMC).

5- Médica-pediatra e professora da Faculdade de Medicina de Campos (FMC)

\section{RESUMO}

Objetivo: Indicar os principais sinais de Transtorno do Espectro do Autismo (TEA) em crianças de zero a três anos. Método: Revisão bibliográfica e entrevista a cinco responsáveis por crianças com diagnóstico de Autismo. Resultados: As cinco entrevistadas relataram ter observado algo de diferente no desenvolvimento das crianças desde os primeiros meses de vida. Discussão: A dificuldade no diagnóstico, antes dos 36 meses de idade se deve, em parte, à falta de conhecimento, pelos profissionais, sobre marcos do desenvolvimento típico. Conclusão: Pais e cuidadores devem estar atentos a atrasos no desenvolvimento infantil, principalmente na interação social e na linguagem pré-verbal, para intervirem precocemente. Palavras-chave: Transtorno do Espectro do Autismo; Diagnóstico Precoce; Intervenção Precoce.

\begin{abstract}
Objective: Indicate the main signs of Autism Spectrum Disorder in children from zero to three years. Method: Bibliographic review and interview of five responsible for children diagnosed with Autism. Results: The five people interviewed reported observing something different in the development of children from the first months of life. Discussion: Difficulty in diagnosis before 36 months of age is partially due to the lack of knowledge by professionals about typical developmental milestones. Conclusion: Parents and caregivers should be aware of delays in child development, especially in social interaction and in preverbal language, to intervene early.

Keywords: Autism Spectrum Disorder; Early Diagnosis; Early Intervention
\end{abstract}




\section{INTRODUÇÃO}

De acordo com o DSM5 (Manual do Diagnóstico e Estatístico de Transtornos Mentais), publicado em 2013, o termo Transtorno do Espectro do Autismo (TEA) substitui a nomenclatura de Transtornos Globais do Desenvolvimento (TGD), adotada no DSM-IV-TR (ASSUNÇÃO JÚNIOR, 2015). As manifestações comportamentais que definem o TEA incluem comprometimentos qualitativos do desenvolvimento sociocomunicativo, presença de comportamentos estereotipados e um repertório restrito de interesses e atividades. Tais sintomas, manifestados conjuntamente,limitam ou dificultam o funcionamento diário do indivíduo.

A manifestação do TEA ocorre precocemente e as dificuldades apresentadas tendem a comprometer o desenvolvimento da criança, ao longo da vida, com grande variabilidade na intensidade e forma de expressão da sintomatologia, nas áreas que definem o seu diagnóstico (ASSUNÇÃOJÚNIOR, 2015). Esse transtorno é definido como uma síndrome comportamental complexa com múltiplas etiologias, com a combinação de fatores ambientais e genéticos. Como até o momento as bases biológicas desse transtorno não foram ainda completamente determinadas, a identificação e o diagnóstico baseiamse nos comportamentos apresentados pela criança e na história do seu desenvolvimento.

A identificação dos primeiros sinais de TEA pode ocorrer já nos primeiros três anos de vida da criança. Cuidadores, pais e profissionais da saúde e da educação são capazes de apontar alterações comportamentais nas crianças antes de as mesmas completarem 12 meses de idade. A identificação e a avaliação precoces destes sinais permitem o direcionamento de intervenções e possíveis ganhos significativos ao desenvolvimento da criança.

A incidência de Autismo é maior em meninos do que em meninas, com razão de quatro meninos para uma menina (CAMINHA,2016, p. 34).O aumento de pessoas diagnosticadas com TEA no mundo é significativo, porém isto não se deve, unicamente, ao aumento da prevalência do transtorno. Este crescimento pode estar relacionado à adoção de definições mais amplas para o Autismo,que é considerado como um espectro de condições; à maior conscientização entre os clínicos e na comunidade, em relação às diferentes manifestações do transtorno; à compreensão sobre a importância da identificação precoce e a intervenção que potencializam um desfecho positivo.
Um diagnóstico precoce é de extrema importância, pois aumenta a possibilidade de a criança se beneficiar dos efeitos da intervenção e dos pais em receberem orientações adequadas para melhor evolução do tratamento. Um número cada vez maior de profissionais tem defendido que a forma mais adequada de se estabelecer o diagnóstico do Autismo é a interdisciplinar, incluindo na equipe pelo menos um neuropediatra e um psicólogo especialista em distúrbios do desenvolvimento. (SILVA, MULICK, 2009).

É notória a importância da intervenção precoce conceituada como um sistema coordenado de serviços com o objetivo de promover o desenvolvimento da criança de zero a cinco anos de idade. Dentre as metas desse sistema estão a estimulação das competências da criança, visando minimização de suas incapacidades e o empoderamento, ou fortalecimento, familiar. (BYINGTON,WHITBY,2011).O modelo da intervenção precoce está ancorado na ideia de que as crianças com deficiência e outras necessidades especiais diferem, de algum modo, daquelas com desenvolvimento típico (MENDES, 2010; NUNES, 1995). Essas diferenças individuais geram demandas de oferta de diversas modalidades de serviços de atendimentos específicos e interdisciplinares.

O modelo interdisciplinar de avaliação é essencial para identificar o foco e o contexto da intervenção. Quando as práticas são focadas nas demandas da criança, depreende-se "como, onde e quando" ocorrerá a prática intervencionista. de(NUNES, RODRIGUES ARAÚJO, 2014). É de suma importância a participação da família em todo processo de intervenção. Os pais são coterapeutas de seus filhos.

\section{A criança com TEA: sinais precoces}

Desde o nascimento, os bebês com desenvolvimento típico se orientam mais para pessoas do que para objetos e se orientam mais para a mãe do que para outros adultos não familiares, além de possuírem, desde muito cedo, comportamentos sociais que podem influenciar os adultos, diferentemente das crianças com sinal de risco para TEA, que apresentam um comprometimento qualitativo no desenvolvimento sociocomunitativo. A não resposta às tentativas de interação é crucial para o diagnóstico do referido transtorno.

Algumas outras características são observáveis em crianças com suspeita ou com diagnóstico de TEA. Dentre elas, destacam-se :dificuldades em mudar de atividades, mantendo-se numa mesma tarefa por um 
período excessivo de tempo; birras e comportamentos heteroagressivos (morder, arranhar, dar pontapés, etc.) e autoagressivos (bater com a cabeça, morder-se, bater nela própria), interesses específicos por determinados objetos ou temas, estereotipias e comportamentos nãofuncionais e atípicos (como enfileirar um número de objetos sempre da mesma maneira e de forma repetitiva), manuseio de partes de objetos, não envolvimento e partilha em brincadeira social, inabilidade em reconhecer emoções, disfunções na atenção, labilidade afetiva, alteração na prosódia, na entonação, na velocidade, no ritmo,vocabulário restrito, emissão de frases aprendidas e usadas fora de contexto e de forma repetitiva e ecolalia imediata ou tardia (LAMPRÉIA, 2007).

Crianças com Autismo não desenvolvem redes neuronais ou sistemas de atenção direcionados para os eventos sociais. Sabe-se que, quanto maior a divergência entre uma criança com Autismo e outra com desenvolvimento típico, mais divergentes serão os padrões de resposta neuronais. Quanto mais ativa e efetivamente envolvida a criança estiver com o mbiente maiores serão as possibilidades de aprendizagem. Adicionalmente, crianças com TEA exibem deficiência no processamento de informação sensorial proveniente de determinados estímulos externos, interpretando-os de maneira bastante diversa das demais crianças. Assim a hipersensibilidade aos estímulos sensoriais são observadas em diferentes graus, como fixar o olhar em movimentos, em luzes e água escorrendo, jogar areia entre os dedos das mãos de forma repetida, exibir atração ou retração a sons, luzes, texturas, cheiros. (WING,GOLD,1979; SAHLEY, PANKSEPP,1987).

Nos primeiros anos de vida, é possível observar nas crianças com Autismo atraso nas aquisições do desenvolvimento global e ausência de comportamentos esperados para a idade cronológica. Com efeito, entre zero e três anos, é possível perceber distúrbios dos multissistemas do desenvolvimento, salientando-se as alterações dos sistemas de regulação do organismo. Muitos pais percebem que algo não vai bem no desenvolvimento do seu filho, porém, com frequência, escutam de profissionais de saúde a resposta: "espere e observe". Sem dúvida, esta não seria a melhor resposta. Há a necessidade do monitoramento do desenvolvimento da linguagem e do comportamento da criança de risco, para que se identifiquem precocemente atrasos ou desvios. Mesmo diante de tantos estudos e escalas para avaliação de Autismo, o olhar clínico é de maior importância entre zero e três anos, pois as escalas frequentemente não se mostram sensíveis na avaliação de bebês e crianças muito jovens. (VOLKMAR, BERGMAN, 1988).

A observação de vídeos caseiros de crianças que tiveram posteriormente diagnóstico de Autismo impulsionaram os estudos sobre bebês de risco (WING, 1979). Estudos realizados a partir de análise de videoteipes gravados no primeiro ano de vida apontam para prejuízos na capacidade de atenção compartilhada, aos 12 meses. Esta refere-se à capacidade humana para coordenar a atenção social, envolvendo também dificuldade de olhar para os outros, de estabelecer a alternância de olhares. Então, nos primeiros anos de vida as principais áreas do desenvolvimento a serem consideradas são a comunicação social e a interação. Entrevistas com os pais são outra fonte importante de informação, bem como a observação direta da criança.

A atenção a alguns sinais pode ajudar a discriminar o Autismo do atraso no desenvolvimento ou do desenvolvimento normal. No primeiro ano de vida são: alteração na interação social; falta do sorriso social; falta de expressão facial adequada; hipotonia, atenção pobre. No segundo ano de vida é observada ausência de respostas de olhar para outras pessoas e de mostrar ou apontar objetos. Quando o fazem, a comunicação é apenas protoimperativa, ou seja, como forma de solicitar acesso a objetos distantes, mas raramente tem função protodeclarativa, ou seja, como modo de afetar as percepções e emoções do interlocutor. Nessa idade, a criança de risco para Autismo não atende ao ser chamada pelo nome, não partilha atenção, não exibe gestos funcionais, apresenta dificuldade em expressar emoções (ADRIEN et al,1993).

Schwartzman (2011) buscou parâmetros diferenciais entre crianças autistas com atraso intelectual e crianças somente com atraso intelectual, descrevendo alguns critérios para o diagnóstico de Autismo em crianças entre 8 e 19 meses de idade. Os sinais básicos destacados por ele foram: ausência no olhar para o outro; não olhar para a face do outro e sorrir; não reagir ao ser chamado pelo nome.

A dificuldade no diagnóstico ocorre, muitas vezes, devido à falta de conhecimento sobre o desenvolvimento típico da comunicação em crianças, o qual prevê a atenção compartilhada (gestos, olhares e comentários espontâneos que visam compartilhar a curiosidade sobre o que ocorre ao redor), o balbucio aos 12 meses, a produção de palavras isoladas aos 16 meses, e a combinação de duas palavras com finalidade comunicativa e presença de brincadeiras de 
faz-de-conta aos 18 meses. Segundo Tomasello (2005) a maioria das crianças com Autismo dificilmente apresenta engajamento colaborativo, bem como pouco participa de atividades culturais e simbólicas. As perdas nas habilidades verbais, comunicativas e sociais, em qualquer idade são sinais preocupantes e requerem atenção (BARBARESI et al, 2006). No caso do Autismo, as variações de linguagem podem variar de acordo com a severidade do quadro clínico: em torno de $30 \%$ das crianças não vão falar (KLIN, 2006) e frequentemente exibem dificuldade de compreensão da linguagem falada (HALL, 2012).

Estudos atuais demonstram que crianças com TEA dificilmente recebem o diagnóstico antes dos 5 anos, ocorrendo com mais frequência quando atingem a idade escolar. Quatro fatores podem determinar o atraso na realização do diagnóstico precoce: 1) a variabilidade na expressão dos sintomas do TEA; 2) as limitações da própria avaliação de profissionais que trabalham com a idade pré-escolar, uma vez que essa população demanda instrumentos específicos e sensíveis aos comportamentos sociais mais sutis e próprios dessa faixa etária; 3)a falta de profissionais treinados/habilidades para reconhecer as manifestações precoces do transtorno; e 4) a escassez de serviços especializados (BOSA, 2002).

A demora na detecção das primeiras dificuldades no comportamento da criança pode retardar na busca pela ajuda profissional, na realização do diagnóstico e principalmente na intervenção dificultando a evolução da criança antes dos 5 anos. (LAMPRÉIA, 2007).

Considerando o acervo de pesquisas apresentado acima, o objetivo do estudo foi verificar como pais de crianças pequenas relatam a observação de sinais de risco para TEA nos primeiros meses de vida da criança,bem como ocorreram a busca e o encontro de atendimento especializado para seus filhos. Método

Participantes: cinco responsáveis (quatro mães e uma avó) por crianças diagnosticadas com Autismo, atendidas pelos profissionais do Ambulatório Interdisciplinar do Hospital dos Plantadores de Cana. Elas são: Marisa, mãe de Roberto, atualmente com 3 anos mas diagnosticado aos 2 anos; Tânia, mãe de Pedro, de 4 anos e diagnosticado aos 18 meses; Sonia, mãe de Lucas, de 7 anos, diagnosticado aos 4 anos ; Olivia, mãe de Ana de 8 anos, diagnosticada aos 5 anos e 2 meses; Janaina, avó de Augusto, de 11 anos, diagnosticado aos 5 anos. Todos os nomes são fictícios. Essas participantes foram selecionadas dentre as famílias atendidas neste Ambulatório, por meio de sorteio.

Local e instrumentos: o estudo foi conduzido no Ambulatório Interdisciplinar da Pediatria do Hospital dos Plantadores de Cana, em Campos dos GoytacazesRJ. Para a coleta de dados foi empregada uma entrevista semiestruturada acerca da percepção dos familiares dos sinais de Autismo em suas crianças. $\mathrm{O}$ roteiro da entrevista está no apêndice 1. Este roteiro foi elaborado a partir de estudo bibliográfico e foi organizado em seis questões abertas para que os pais ou responsáveis pudessem responder, expondo suas observações e justificando-as, dissertativamente.

Procedimentos: o entrevistador iniciou esclarecendo aos participantes sobre o objetivo da pesquisa. As entrevistas, filmadas e posteriormente transcritas verbatim, foram realizadas individualmente nos meses de novembro e dezembro de 2016 e tiveram a duração média de 30 minutos. As dúvidas existentes foram solucionadas no momento da realização das entrevistas, durante as quais não houve ocorrências que prejudicassem a sua realização.

\section{RESULTADOS}

Quatro entrevistadas tinham entre vinte e nove e trinta e cinco anos e uma, cinquenta e nove anos de idade. Das cinco entrevistadas, três se separaram de seus cônjuges após o nascimento do filho especial. Quatro das entrevistadas recebiam até dois salários mínimos e eram responsáveis pela manutenção de seus lares; apenas uma recebia cerca de três salários mínimos.

Corroborando os achados da literatura (BOSA, 2002), as cinco entrevistadas relataram que observaram algo de diferente no desenvolvimento das crianças desde os primeiros meses de vida: ausência de contato visual, choro constante, sorriso não responsivo e intolerância ao barulho, antes de 12 meses de idade. Três participantes relataram que tiveram "bebêsmodelo", que jamais choravam e sempre preferiam estar sós; duas relataram que seus bebês choravam muito, quase "o tempo todo"; uma relatou que seu bebê não se aninhava no colo e nunca sorria diante de carícias ou "gracinhas" feitas pelos adultos.

$\mathrm{O}$ atraso na fala e no andar, em torno de 18 meses, levaram-nas a procurar especialistas, como pediatras, para que esclarecessem as possíveis causas deste desenvolvimento atípico. Em todos os casos, os primeiros profissionais procurados consideraram um atraso normal, pois "cada criança tem o seu ritmo"; "calma, ele vai progredir..., "você está vendo coisa 
demais...(sic)". Tal situação gerou um sentimento de desamparo nas responsáveis, pois sentiam que suas preocupações não eram levadas a sério, principalmente pelos pediatras, os primeiros procurados pelas famílias.

Três mães disseram ter se sentido incompetentes em relação à criação e cuidados dispensados aos filhos. Uma das mães relatou "sentir-se culpada, por achar que não fez o vínculo primário com seu bebê". Segundo ela, um pediatra consultado, havia dito que esta seria a causa do Autismo. Após a entrevista, a entrevistadora conversou com a mesma, mostrando ser este um equívoco. De fato, existe consenso entre os especialistas de que o Autismo é decorrente de disfunções do sistema nervoso central (SNC), que levam a uma desordem no padrão do desenvolvimento da criança (SILVA, MULICK,2009, p 121). Desta forma, atualmente, nenhum profissional qualificado propõe a existência de "uma causa" para o Autismo. O máximo que se tem proposto é a identificação de fatores genéticos e biológicos que parecem contribuir para a exibição de certos sintomas ou características particulares, ou de subtipos específicos dentro da população geral de indivíduos com TEA.

Foi constatado que as responsáveis dedicavam todo o seu tempo aos cuidados com estes menores, abdicando da vida profissional em busca de terapias para que assegurassem melhor qualidade de vida aos pequenos. As cinco responsáveis, diante dos problemas de relacionamento social que se acentuavam, além de alguns comportamentos agitados, "agressivos" e do atraso na linguagem, continuaram na busca de um diagnóstico. Elas encontraram posteriormente esclarecimento acerca de suas observações com profissionais que conheciam sobre Autismo e estabeleceram com os mesmos uma relação de confiança. Estes profissionais eram dois neuropediatras, um pediatra especialista em desenvolvimento infantil, um psiquiatra infantil e uma psicopedagoga, que as encaminharam para uma equipe multi e interdisciplinar, visando tratamento para o transtorno no Ambulatório Interdisciplinar do Hospital dos Plantadores de Cana.

É notório que os custos humanos, sociais e financeiros decorrentes das necessidades dos indivíduos com TEA costumam ser bastante elevados. Dados epidemiológicos de países desenvolvidos revelam que crianças com TEA frequentam nove vezes mais os serviços de saúde do que crianças com outros problemas médicos e três vezes mais que crianças com deficiência mental (Duarte et al., 2016). Desta forma, podemos considerar as implicações financeiras aos serviços de saúde e de educação, e aos programas de intervenção precoce. Sabe-se que os procedimentos relativos ao diagnóstico de TEA, bem como à assistência são complexos, por isso grande parte da população ainda não esteja recebendo tratamento adequado no Brasil.

Foi ressaltada, por todas as responsáveis, a importância do conhecimento do desenvolvimento típico da criança pelo profissional que lida com ela, além da capacidade de observação das peculiaridades de cada uma. Este conhecimento foi apontado como principal fator, que pode favorecer melhor prognóstico no tratamento, pois leva à identificação dos primeiros sinais e, consequentemente, à intervenção precoce nos primeiros anos de vida, período em que há maior neuroplasticidade cerebral, e assim, maior chance de êxito nas intervenções.

Todas as participantes reafirmaram que as terapias com fonoaudiólogos, psicólogos, terapeutas ocupacionais e psicopedagogos possibilitaram desenvolvimento significativo nas crianças, em especial, na melhora da comunicação e no relacionamento social. Quatro das entrevistadas citaram a importância de encontrarem profissionais que as ajudaram na educação de seus filhos. Todas citaram serem necessárias redes de apoio e grupos de pais, para troca de experiências e ajuda mútua.

Dentre os aspectos citados pelos participantes como melhora no tratamento, estão: a) Marisa, mãe de Roberto: melhor interação social na escola e com coleguinhas e parentes, tendo deixado de se esconder atrás das portas e embaixo da Cana: desenvolvimento da linguagem oral, agora falando frases com duas palavras ("dá bola", "quer água") e cantando na Igreja que frequenta; menor incômodo diante de sons altos;diminuição do apontar imperativo; b) Tânia, mãe de Pedro: deixou de brincar com partes do brinquedo, brincando agora de forma funcional; diminuição da ecolalia tardia; melhor participação nas atividades escolares; c) Sônia, mãe de Lucas: melhora na "alternância de turnos"; ampliação do vocabulário; menor irritabilidade e agitação psicomotora; aumento do repertório de interesses, cujos focos eram apenas os "mamutes e o Ben 10"; passou a atender pelo nome, quando chamado; diminuição de comportamentos autolesivos; d) Olívia, mãe de Ana: passou a entender e respeitar ordens simples; diminuiu movimentos estereotipados, hoje imita (solta beijos, dá tchau), responde com sorriso social, segue o olhar do adulto; e) Janaína, avó de Augusto:passou a brincar com os brinquedos de forma funcional; diminuiu a irritabilidade 
diante de determinados sons, como foguetes, buzina de automóveis; está alfabetizado; tem participado de atividades grupais, como jogos e excursões escolares;tem interesse por livros e jogos, como jogo da memória e caça-palavras, houve redução dos movimentos estereotipados.

"Tudo é passo a passo. Temos que entrar no mundo deles para eles entrarem no nosso." - relatou Janaína, a entrevistada mais idosa do grupo. Esta senhora sempre ajuda as demais com palavras de incentivo e bom ânimo na sala de espera, segundo as demais entrevistadas.

As participantes relataram igualmente que neste Ambulatório, onde foi realizada a pesquisa, foram criados novos sentimentos de esperança e confiança de que essas crianças poderão vir a ser mais independentes e com melhores perspectivas na vida futura. Todas as crianças estão sendo atendidas no referido Ambulatório há mais de um ano; sendo que as três mais velhas, de 7, 8 e 11 anos, estão inseridas há três anos nas terapias.

Identificamos o aspecto "adquirir autonomia" como a principal preocupação no relato de quatro das responsáveis, quando pensam em como estarão seus filhos/neto na vida adulta, se conseguirão trabalhar e se constituirão família. Também foram citadas por três mães as dificuldades encontradas para a inclusão escolar de seus filhos. Segundo Gomes e Nunes (2014 p.145), a tríade sintomatológica que caracteriza o perfil da pessoa com Autismo não pode ser ignorada no contexto da escola. As especificidades da síndrome costumam interferir no aprendizado e na inclusão social dessas crianças em escolas. Também, a proximidade física com os colegas, a dificuldade em aprender e respeitar regras sociais, a falta de compreensão de instruções verbais ou a incapacidade em utilizar a linguagem falada podem representar grandes dificuldades e desafios para esse grupo de crianças.

\section{CONCLUSÕES}

$\mathrm{O}$ acervo de estudos revisados aponta para a importância do conhecimento sobre o desenvolvimento típico em crianças entre zero e três anos, que possa favorecer a identificação precoce de possíveis alterações no desenvolvimento relacionadas com os sinais do TEA por pais e profissionais da saúde e da educação. Os pais e cuidadores podem ser, e geralmente o são, os primeiros identificadores dos sinais de atrasos no desenvolvimento de seus filhos, $o$ que é fundamental para a busca do auxílio dos profissionais da saúde. O profissional deve levar em consideração essas observações, investigando as possíveis causas deste atraso, possibilitando a intervenção precoce em serviços especializados.

Faz-se necessário que os pediatras, diante de relatos parentais, procurem conhecer o desenvolvimento social das crianças, buscando detalhes e exemplos, principalmente se a queixa principal estiver relacionada ao atraso do desenvolvimento das linguagem pré-verbal e oral. Torna-se importante observar tais sinais na primeira infância, pois constituem um primeiro passo, direcionando ao diagnóstico precoce, possibilitando assim a criança se beneficiar dos efeitos da intervenção.

Quando confirmado o diagnóstico de Autismo os profissionais precisam, então, determinar quais encaminhamentos ainda se fazem necessários. Isso inclui encaminhamentos para terapeutas ocupacionais, fisioterapeutas, fonoaudiólogos, pedagogos, psicólogos, neurologistas, geneticistas e/ou para profissionais que lidam com problemas alimentares e/ou de sono.

É fundamental, também, que a criança e a sua família sejam encaminhadas para programas educacionais específicos, incluindo cursos e programas que auxiliem os pais no enfrentamento dos problemas decorrentes do TEA. O foco na família refere-se ao fornecimento de suportes para que os cuidadores possam dispor de energia, tempo, saúde física e mental, além de conhecimentos para oportunizarem aprendizagens indispensáveis ao desenvolvimento da criança.

Com a recente Lei $\mathrm{n}^{\circ} 13.438$ (D.O de 26 de abril de 201, seção 1), torna-se obrigatória, pelo Sistema Único de Saúde (SUS), a aplicação de protocolo para avaliação de sinais de risco para o desenvolvimento psíquico da criança nos dezoito primeiros meses de vida, em consulta pediátrica, o que deve facilitar a detecção precoce dos sinais de risco para Autismo.

Assegurar que todas as diferentes áreas em que a criança apresente problemas sejam efetivamente investigadas e tratadas é de máxima importância. Avaliações psicológicas devem ser recomendas anualmente, para que se possa acompanhar o progresso da criança ao longo dos anos e ajudar na revisão dos programas e intervenções recebidos por ela.

Também, pode-se concluir que: a) o diagnóstico precoce de Autismo pode ser hipotetizado dentro dos primeiros 2 anos de vida; b)ainda que o diagnóstico não seja conclusivo, intervenções visando minorar os comprometimentos nas áreas atingidas devem ser efetuadas; c) com intervenções adequadas, as seguintes funções básicas podem ser desenvolvidas: contato visual, atenção compartilhada e imitação. 


\section{REFERÊNCIAS BIBLIOGRÁFICAS}

ADRIEN, J. L., et al. Blind ratings of early symptoms of autism based upon family home movies. Journal of American Academy of Child and Adolescent Psychiatry, v. 32, n. 3, p. 617-626, 1993.

Assunção Júnior, Francisco B, et al. Autismo Infantil: Novas Tendências e Perspectivas.2. ed. São Paulo: Atheneu, 2015.

BARBARESI, W. J. et al. Autism: a review of the state of the science for pediatric primary health care clinicians. Arquive of Pediatric and Adolescent Medicine, v. 160, p. 1167-1175, 2006.

BOSA, C. Atenção compartilhada e identificação precoce do Autismo. Psicologia, Reflexão e Crítica, v.. 15, n 1, p. 77-88, 2002.

BYINGTON, T., WHITBY.P. Empowering Families During the Early Intervention Planning Process. Young Exceptional Children, v. 14, p. 44-56. 2011. Disponível em: <http://dx.doi.org/10.1177/1096250611428878>Acesso em $1^{\circ}$ de março de 2017.

CAMINHA, Vera Lúcia; ALVES, Priscila Pires (orgs); Autismo Vivência e Caminhos. Disponível em: <http:// openaccess.blucher.com.br/article-list/Autismo-92/list\#articles>Acesso em: 03 de novembro de 2016.

DUARTE, Cíntia Perez et al. Diagnóstico e Intervenção precoce no transtorno do espectro do Autismo: relato de um caso. In:CAMINHA, Vera (org). Autismo: vivências e caminhos. São Paulo: Blucher, 2016.

GOMES, Rosana Carvalho; NUNES, Débora R. P.. Interações comunicativas entre uma professora e um aluno com Autismo na escola comum: uma proposta de intervenção. Educ. Pesqui., São Paulo, v. 40, n. 1, p. 143-161, Mar. 2014.Disponível em: <http://www.scielo.br/scielo.php?script=sci_arttext\&pid=S151797022014000100010\&lng=en\&nrm=iso >. Acesso em: 18 de abril de 2017.

HALL, Laura J. Autism Spectrum Disorders: from theory to practice. UpperSaddle River, NJ: Merrill Prentice Hall, 2012.

KLIN, Ami. Autismo e Síndrome de Asperger: uma visão geral. Revista Brasileira de Psiquiatria, v. 28, supl. 1, p. 3-11, 2006.

LAMPRÉIA, C. A perspectiva desenvolvimentista para a intervenção precoce no Autismo. Estudos de Psicologia, v. 24, n. 1, 2007, p. 105-114.

MENDES, E. G. Inclusão marco zero: começando pelas creches. Araraquara, SP: Junqueira \& Marin, 2010.

NUNES, Débora R. P., ARAÚJO, Eliana R. Autismo: a educação infantil como cenário de intervenção. Education Policy Analysis Archives/Archivos Analíticos de Políticas Educativas. Arizona, Estados Unidos. v. 22. P. 1-14. 2014. Disponível em em:<http://www.redalyc.org/articulo.oa?id=275031898092> Acesso em 24 de abril de 2017. NUNES, L. R. Educação precoce para bebês de risco. In: RANGE, B.(Org.). Psicoterapia comportamental e cognitiva. Campinas: Psy, 1995, p. 121-132.

SAHLEY,T.L.; PANKSEPP, J. Repetitive Behaviour in Autism: A Review of Psychological Research. Journal of Autism and Developmental Disorders. 17: 201, 1987.doi:10.1007/BF01495056.

SCHWARTZMAN, José Salomão; ARAÚJO, Ceres Alves. Transtornos do espectro do Autismo-TEA. São Paulo: Mennon, 2011.

SILVA, Micheline; MULICK, James A.. Diagnosticando o transtorno autista: aspectos fundamentais e considerações práticas. Psicol. cienc. prof., Brasília , v. 29, n. 1, p. 116-131, 2009. Disponível em:<http://www.scielo.br/ scielo.php?script=sci_arttext\&pid=S1414-98932009000100010\&lng=en\&nrm=iso $>$ Acesso em 19 de abril de 2017. TOMASELLO et al. Understanding and sharing intentions: the origens of cultural cognition. Behavioral and Brain Sciences, v. 28, p. 675-735, 2005.

WING, L.; GOULD, J. Severe impairments of social interaction and associated abnormalities in children: Epidemiology and classification. Journal of Autism and Developmental Disorders,v. 9, p. 11-29, 1979.

VOLKMAR, F.R.; BERGMAN, J.; COHEN,D.J. et al. DSM III and DSM III-R diagnoses of autism.AmJ Psychiatry.v. 145 p. 1404-1408, 1988. 